\title{
Multifragmentation in the Intranuclear Cascade - Percolation Approach
}

\author{
J. Cugnon ${ }^{1}$ and C. Volant ${ }^{2}$ \\ ${ }^{1}$ Université de Liège, Physique Nucléaire Théorique, \\ Institut de Physique au Sart Tilman, Liège, Belgium \\ ${ }^{2}$ DPhN-BE, CEN Saclay, Gif-sur-Yvette, France
}

Received October 18, 1988; revised version August 7, 1989

Results of intranuclear cascade calculations followed by a percolation procedure lead to an encouraging comparison with the experimental mass distributions observed in proton and heavy ion induced reactions in the intermediate energy domain. The predictive power of the model as well as its implications and limitations are examined.

PACS: $25.70 . \mathrm{Np}$

\section{Introduction}

In recent years a large amount of attention has been paid to the study of violent collisions of highly energetic protons and heavy nuclei with target nuclei. The interaction is generally assumed to proceed in two steps: in the fist one, the incident nucleons scatter on the target ones ejecting some of them and leaving a certain amount of energy to the spectator part, which forms rather heavy and excited residues. In the second stage, after escape of the participant nucleons, the residues decay through "normal" evaporation of nucleons, small fragments and lower mass cold residues (or fission) for low primary excitation energies or, at higher excitation energies, disintegrate into more sizeable and numerous fragments of intermediate mass (IMF), accompanied sometimes by rather heavy residues.

The first step can be handled, for instance, by means of the intranuclear cascade (INC) model [1, 2], which has been successfully used for heavy ion reactions, antiproton annihilation on nuclei and protonnucleus reactions, ([3-5] and references therein). With regard to the second stage, the production of IMF's (referred to here as masses typically between 4 and $50 \mathrm{amu}$ ) has been observed with apparently similar characteristics in the high energy domain (e.g., protons up to $350 \mathrm{GeV}$ [6], ${ }^{20} \mathrm{Ne}$ projectiles of $2100 \mathrm{MeV} / \mathrm{u}$ [7]) as well as for heavy ions at low bombarding energy [8]. Several phenomenological descriptions of this second step have been proposed to explain the IMF production [9-21]. The current models differ drastically leading however virtually to the same results. For example the dependence of the IMF mass distribution following a power law $Y(A)=A^{-\tau}$, with $\tau$ ranging from 2 to 3 had been originally put forward as an evidence for a manifestation of a gas-liquid phase transition in nuclear systems $[14,15]$. All the modelizations mentioned above are able to reproduce qualitatively this aspect. Clearly other observables are needed to discriminate between different hypotheses, such as the energy spectrum of the IMF's $[22,23]$ or higher momenta of the mass distributions [24].

Certainly, the first interaction stage is of importance to prepare the phase space on which the second stage will act on to lead to the final (fragmentation) state. In this paper, the dynamical treatment used to describe the first stage of the collision is the INC model which is, in the considered energy range, one of the most sophisticated ones and probably, among these, the most tractable one. Since the INC model is not able by itself to generate clusters, we have to supplement it with some procedure. Here, we propose to adopt a percolation procedure, which from the phase space distribution of nucleons at the end of the cascade will construct clusters. This procedure can be viewed as a generalization of the method proposed in [25], aimed to construct deuteron cross-section from cascade. In simple words, this method views deu- 
teron as a neutron and a proton sufficiently close in phase space. It turned out to be quite successful $[26,27]$ to explain deuteron data in the Bevalac regime [28]. This idea has already been used, when the primary collision stage is described by cruder models [19] when the state at the end of the collision is supposed to be completely at random $[29,30]$.

Therefore, our approach includes detailed dynamics in the first stage and a simple model for the generation of fragments in the second stage. Ambitious approaches exist, which try to include mean field effects as the main agent for the splitting of the systems into fragments. They are very promising, though the proper description of the mean field at the end of the collision process is far from being clarified. Our purpose is therefore to address ourselves the following questions:

(1) Is the INC plus percolation picture a successful one?

(2) Can it reproduce mass yield and parallel momentum distribution?

The paper is organized as follows. In Sect. 2, we described our approach. In Sect. 3, we concentrate on proton-induced data. Section 4 is devoted to $\mathrm{Ne}$ $(250 \mathrm{MeV} / \mathrm{u})+\mathrm{Au}, \quad \mathrm{Ne}(2.1 \mathrm{GeV} / \mathrm{u})+\mathrm{Au}$ and $\alpha$ $(1.05 \mathrm{GeV} / \mathrm{u})+\mathrm{Th}$ data. Section 5 contains the discussion and our conclusion.

\section{The Model}

We use the INC model developed in [2]. The description can be found in $[31,32]$. It is sufficient here to say that particles are followed in phase space and that the present version contains pions and delta's, includes isospin degrees of freedom, Pauli blocking and freezing of the Fermi motion for the spectators.

The cascade process is stopped at some time $t_{0}$ and the percolation procedure is then applied to the space distribution. The time $t_{0}$ is determined as follows. In the energy domain considered here the time evolution of the cumulated number of baryon-baryon collisions has some typical behaviour, described for instance in [2]. It shows a steady increase and then suddenly flattens. We choose $t_{0}$ as the time $\left(t_{c}\right)$ at which this phenomenon occurs. The remaining collisions after the moment of this rather strong change constitute only a small part of the total number of collisions (5-7\% at the most). Moreover, these collisions are soft and do not change the momentum distribution any more. After time $t_{0}$ the system is expanding. Therefore our choice for the time of application of percolation (applied in $\mathbf{r}$-space) may appear quite arbitrary. Of course, it cannot be taken earlier than $t_{c}$, but one may expect that the results of the percolation procedure are sensitive to the time of percolation for values larger than $t_{c}$. Fortunately, it turns out that for a few $\mathrm{fm} / \mathrm{c}$ 's after time $t_{c}$, the system does not yet contain strong $\mathbf{r}-\mathbf{p}$ correlations (with position and momentum vectors, in the c.m. frame, strongly aligned), signalling the steady mutual recession of nucleons in a fully developed free-space expansion. In other words, for this time span, the nucleons tend to escape, but their average relative distance is not yet changing very much.

The percolation procedure is a very standard one for disordered systems. We look for the space distribution of the particles and we decide that two nucleons belong to the same cluster, if their relative distance $r_{i j}\left|\mathbf{r}_{i}-\mathbf{r}_{j}\right|$ is smaller than some "percolation distance" $d_{c}$ :

$r_{i j} \leqq d_{c}$.

Technically, this is realized by constructing the minimum spanning tree $[33,34]$ based on the relative distances ("links") between nucleons. Afterward, the links larger than $d_{c}$ are cut out. The remaining links uniquely determine the clusters. The percolation distance $d_{c}$ is chosen to be $2 \mathrm{fm}$. In the spirit of this paper, this value should be considered as a phenomenological parameter. Nevertheless, this choice is supported by several observations. First, it is given more or less by the average nucleon distance in nuclear matter close to the critical (isothermal spinodal) density, which corresponds to the onset of instabilities in expanding nuclear fireballs $[35,36]$. Second, we checked that it gives roughly the same results as the ones obtained with a (perhaps) more satisfactory percolation in phase space, which would demand

$\frac{r_{i j}^{2}}{r_{0}^{2}}+\frac{p_{i j}^{2}}{p_{0}^{2}} \leqq 1$

where $r_{0} p_{0}=\hbar$, provided $r_{0}$ is chosen close to $d_{c}$. The only difference occurs for deuterons and to a lesser extent for $A=3,4, \ldots$ clusters, which are more abundantly (up to a factor $\sim 1.5-2$ ) produced with (2.1). For heavier clusters, the two procedures yield the same results to a large extent. The percolation in phase space presents in fact some ambiguity because of the different nature of $r$-and $p$-spaces. Instead of (2.2), it was sometimes [17] proposed to choose the following criterion

$r_{i j} p_{i j} \leqq \zeta \hbar$

with $\zeta$ close to unity. This procedure, however, generates very elongated clusters, provided the partner's momenta are sufficiently close to each other. Actually, in [17], an additional recipe was needed to transform these elongated clusters in more compact ones. 
All criteria above will depend upon the time at which they are applied. The following one

$\rho_{i j} \leqq d_{c}$

where

$\vec{\rho}_{i j}(t)=\mathbf{r}_{i j}(t)-\left[\left(\mathbf{r}_{i j}(t)-\mathbf{r}_{i j}\left(t_{0}\right)\right) \cdot \mathbf{p}_{i j}\right] \frac{\mathbf{p}_{i j}}{\mathbf{p}_{i j}^{2}}$

is independent of $t$ for times later than $t_{0}\left(>t_{c}\right)$. It is easy to check that $\rho_{i j}^{2}(t)$ is constant in the absence of collisions. Of course, criterion (2.5) is equivalent to criterion (2.1), since $\vec{\rho}_{i j}$ reduces to $\mathbf{r}_{i j}$ when $t \rightarrow t_{0}$. Owing to (2.5), one can write (2.4) equivalently as:

$p_{i j}^{2} \rho_{i j}^{2}-\left[\left(\mathbf{r}_{i j}(t)-\mathbf{r}_{i j}\left(t_{0}\right)\right) \cdot \mathbf{p}_{i j}\right]^{2} \leqq d_{c}^{2} p_{i j}^{2}$.

This shows that percolation with criterion (2.5) (or (2.1)) differs from percolation in phase space (like (2.3)) by, of course, the non constant value of the r.h.s. but more basically by the removal of the trivial $p_{i j} \cdot r_{i j}$ correlations built in by the free space (no collision) expansion. The fact that $r$ and $p$ spaces do not seem to play the same role in this context ultimately comes from the fact that nuclear interactions are basically described in $\mathbf{r}$ space. For all these reasons we think that criteria (2.1) and (2.4) are acceptable from the physical point of view.

\section{Proton-induced Reactions}

Results of our calculations are compared in Fig. 1 with IMF mass distributions obtained by the Purdue's group with $3.8 \mathrm{GeV}$ protons on a Xe target. The present results using no adjustment of the standard INC input and obtained from one thousand events for each of five impact parameters ranging from 0 to $6 \mathrm{fm}$ reproduce the trends of the experimental mass yield with almost the same quality as the droplet model fit proposed in [37], but also the absolute magnitude quite well. In order to compare with our predictions, we have multiplied the differential production cross-sections of [37] by $4 \pi$, assuming an isotropic production pattern, in agreement with the indications of [39]. The effect of the inclusion of a (simplified) evaporation procedure is shown in Fig. 2. In our approach, the excitation energy of the primordial cluster is solely coming from the random motion of the nucleons in the cluster c.m. frame after removal of the underlying Fermi motion component. The mass yield is only slightly changed by evaporation, acquiring a somewhat steeper sloper. The dependence upon the impact parameter is illustrated by Fig. 3, where we have compared the total yield with the one which comes

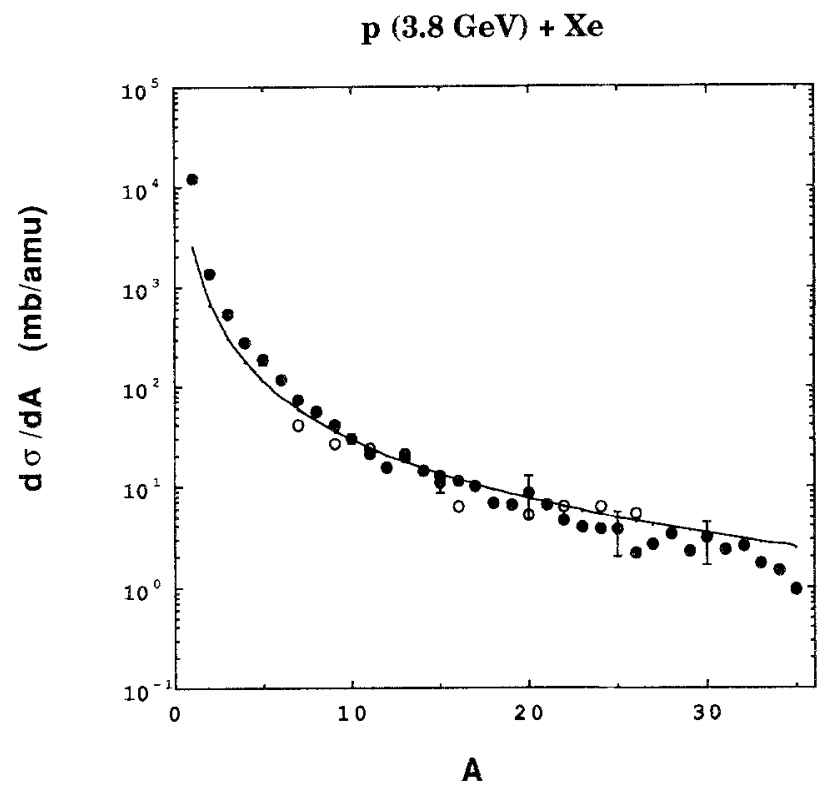

Fig. 1. Mass yield for fragments produced in $p+$ Xe reactions at $3.8 \mathrm{GeV}$. The open dots and the full line represent the data and the fit provided by [37], respectively. The experimental differential cross-sections have been transformed into angle-integrated crosssections, assuming isotropic production (see text). The full dots give our results and the error bars indicate the statistical uncertainty of the calculation

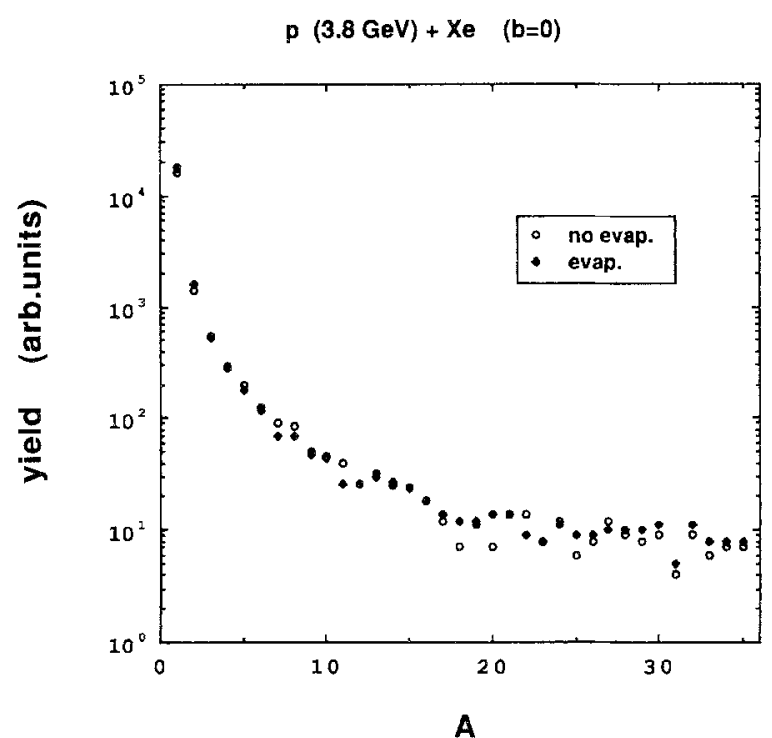

Fig. 2. Comparison of mass yields prior (open dots) and after (black symbols) evaporation for central proton collisions on a Xe target at $3.8 \mathrm{GeV}$. See text

from central $(b=0)$ collisions. In order to make the comparison possible, we have normalized the two curves on the same $A \approx 10$ yield. The inclusion of all impact parameters have roughly the same qualitative effect as the inclusion of the evaporation (steepening of the curve), but quantitatively the evaporation is less important. 


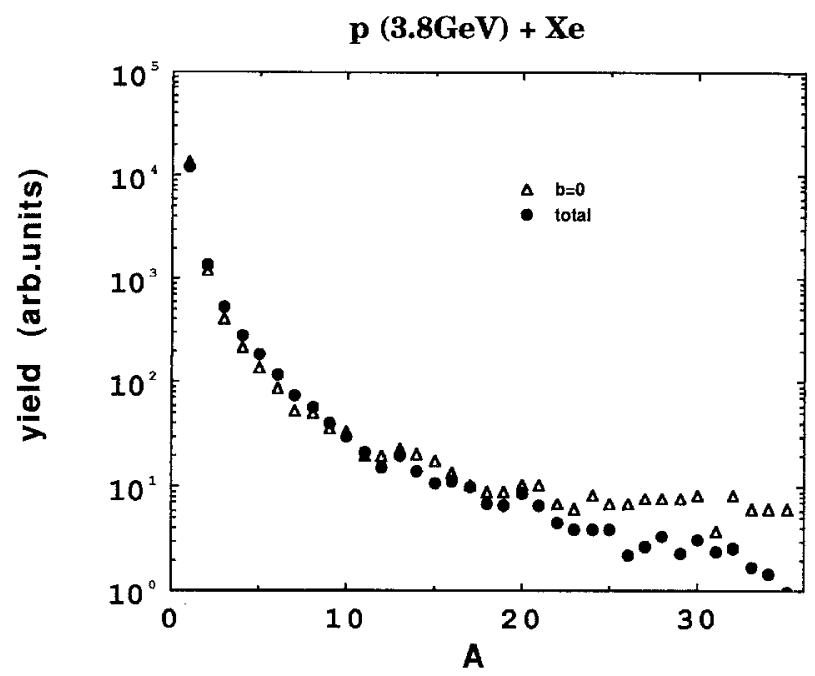

Fig. 3. Comparison of mass yields (in arbitrary units) for purely central collisions (triangles) and for all impact parameters (dots). The two curves are normalized on the same value for $A \approx 10$

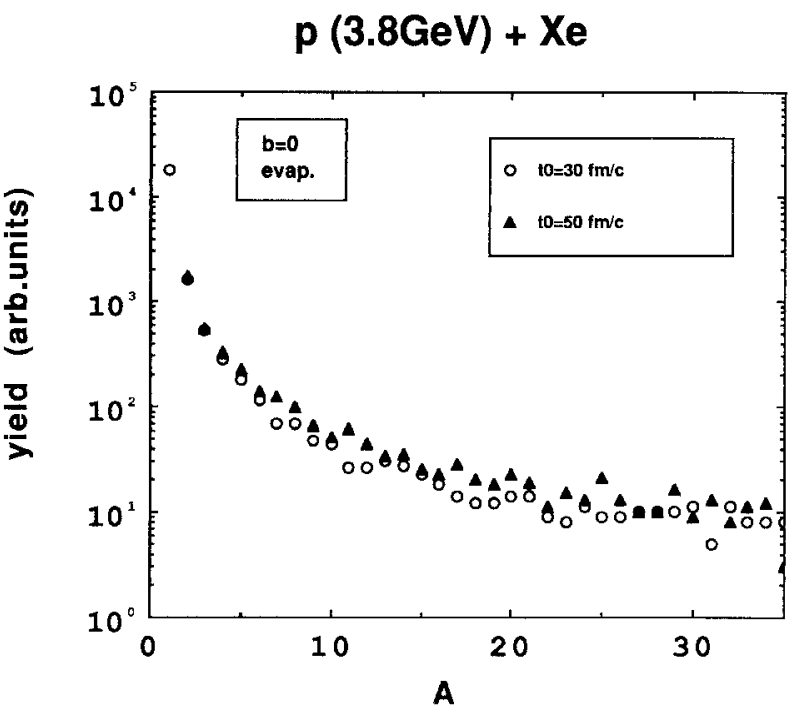

Fig. 4. Central collisions between $3.8 \mathrm{GeV}$ protons on a Xe target: mass yield calculated as explained in the text for $t_{0}=30 \mathrm{fm} / \mathrm{c}$ (open dots) and for $t_{0}=50 \mathrm{fm} / \mathrm{c}$ (black symbols), respectively

We now give a little more detail about the sensitivity of the model. In Fig. 4, we show the mass yield (after evaporation) obtained when stopping the cascade, for $b=0$, at $t_{0}=30 \mathrm{fm} / \mathrm{c}$ and at $50 \mathrm{fm} / \mathrm{c}$, respectively, applying exactly the same percolation procedure with the same $d_{c}$ parameter. As we said in Sect. 2, the cascade + percolation remains rather stable for a long time. To complete the information, at $30 \mathrm{fm} / \mathrm{c}$, $95 \%$ of the cumulated number of collisions have occurred. The conclusion is that during this time span, the collisions are scarce and the $\mathbf{r} \cdot \mathbf{p}$ correlations are

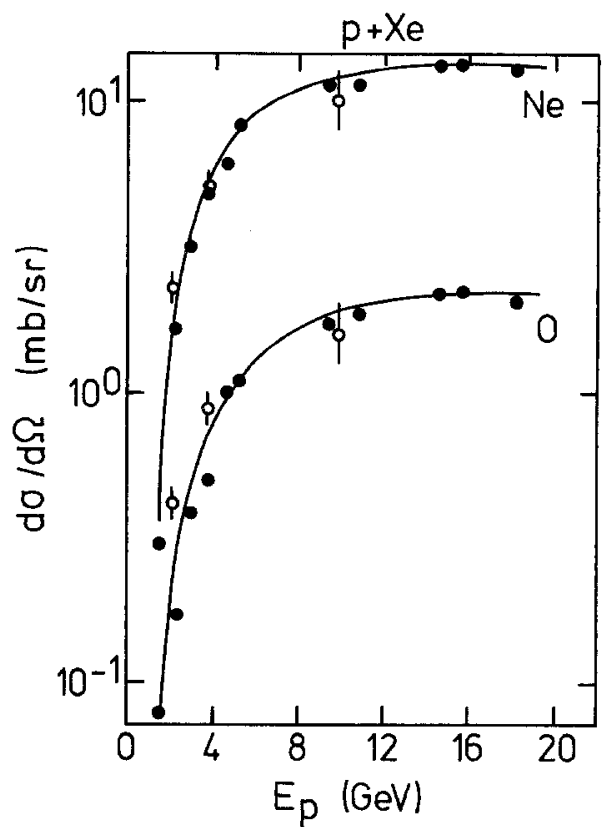

Fig. 5. Excitation function for $\mathrm{O}$ and Ne production in $p$-Xe collisions. The full dots and the curves are the data and simple fits taken from $[38,39]$. The open dots are the results of our calculation

not yet built up. We have to say however that the difference is slightly larger when comparison is made on the primordial cluster yield (i.e. before evaporation): IMF's are produced more abundantly in the percolation at $50 \mathrm{fm} / \mathrm{c}$, but they are less excited. In any case, the difference may be roughly removed by increasing the value of $d_{c}$. To give a more quantitative statement, in that particular case, the yield at $30 \mathrm{fm} / \mathrm{c}$ with $d_{c}=2 \mathrm{fm}$ is equivalent to the one at $50 \mathrm{fm} / \mathrm{c}$ with $d_{c} \approx 2.2 \mathrm{fm}$.

We also calculated the mass spectrum at 2.1 and $10 \mathrm{GeV}$ (for central collisons only, in that last case). For the highest energy, our calculation is only indicative, since the INC model includes inelasticity through single $\Delta$ production. The main reult is contained in Fig. 5, where we show our predictions for $A=16$ and $A=20$ fragments along with the data of $[38,39]$. For making the comparison possible, we have assumed an isotropic production pattern for these fragments, which is presumably legitimate for fragments of this size. For the points at $10 \mathrm{GeV}$, we estimated the total cross-sections for our calculated $b=0$ contribution, assuming the same relationship as at $3.8 \mathrm{GeV}$. Taking account of the fact that the experimental data refer to $Z=8$ and $Z=10$ fragments and that the corresponding errors are of the order of $10-12 \%$ [38], one can say that we reach a fair agreement in the description of the excitation functions. Note however that the calculated yield at $10 \mathrm{GeV}$ is somewhat smaller than the experimental data. This is interesting since the target excitation energy and therefore its fragmen- 
tation crucially depends upon the detail of the inelastic processes in this energy range [5].

We also looked at the "apparent exponent" $\tau^{\prime}$ present in the fitting of the $(A \leqq 40)$ mass yield

$$
Y(A) \propto A^{-\tau^{\prime}} .
$$

The values extracted from our calculation show a similar trend as those obtained experimentally [38] although they are substantially larger: by $\sim 0.2-0.4$, when the fit is restricted from $A=6$ to $A=26$ as in $[37,38])$. We do not want to comment on the physical meaning of the numerical values for this parameter. However, it is expected that in our approach this parameter $\tau^{\prime}$ is directly related to $d_{c}$. A smaller $d_{c}$ will decrease $\tau^{\prime}$. We plan to investigate this point in more detail in the future.

\section{The Heavy Ion Case}

Results for heavy ion induced reactions are shown in Fig. 6 for the two extreme measured bombarding energies for the $\mathrm{Ne}+\mathrm{Au}$ system. Here, we restrict to central $(b=0)$ collisions, because calculations leading to a reasonable statistics for IMF's are very time-consuming. To give an idea, the average $A=1$ (proton) multiplicity is about 60 , while the one for $A \approx 16$ is of the order of $0.01-0.02$. Therefore one needs in principle $9 \times 10^{6}$ more events to achieve a statistics of $A=16$ fragments e.g. as good as the one of the inclusive proton crosssection. Needless to say that, for the moment, it is very hard to have good estimates of observables but the mass yield. Figure 6, which includes evaporation of the fragments, shows a nice agreement with the experimental data, taking account that no adjustment is done. The somewhat steeper slope obtained in the calculation can be attributed to the choice of $d_{c}$. It is expected that a better agreement could be obtained by a slight readjustment of this parameter.

For the same reasons as those mentioned above, we did not calculate many impact parameters. However, we checked that the good agreement displayed in Fig. 6 is not destroyed by large impact parameter, by making an explicit calculation for an intermediate impact parameter at $2.1 \mathrm{GeV}$. As can be seen in Fig. 7, the mass spectrum of the IMF's is roughly the same as for central collisions (at least for $A \geqq 10$ ). Of course, the light fragment $(A \leqq 4)$ yield does depend much more strongly on the impact parameter, a results known for a long time [41].

The present approach also predicts the survival of heavy residues depending upon energy and projectiles. One example is shown in Fig. 8 for the $\alpha+$ Th system where the calculated total primary mass distri-

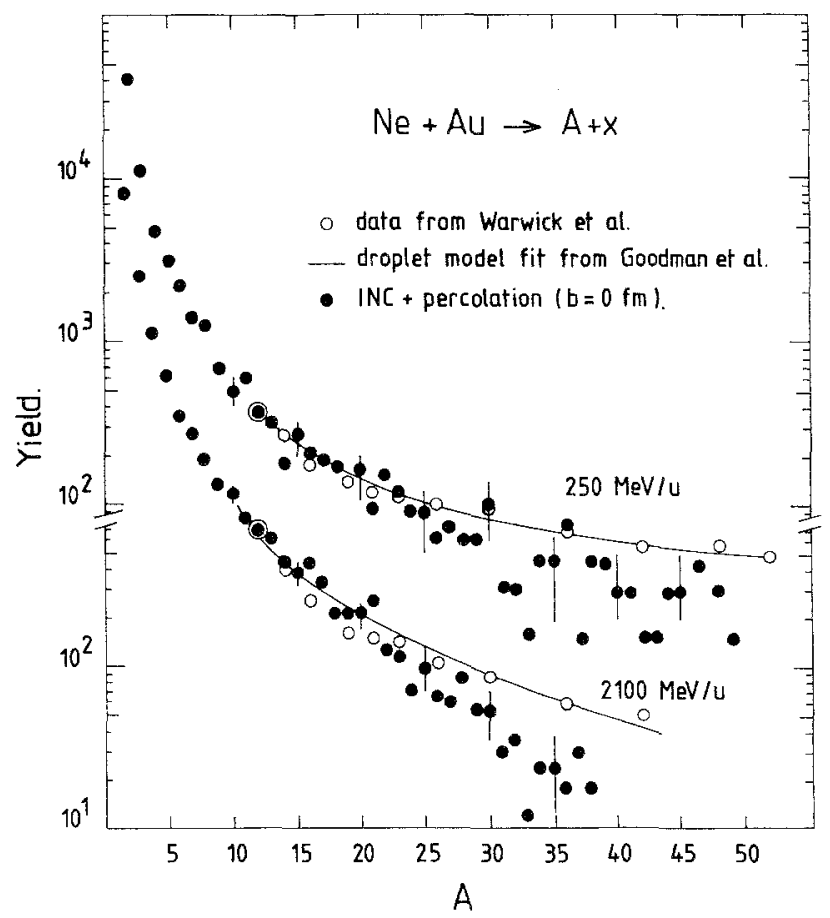

Fig. 6. Open dots: experimental mass yield from [7]. Full curves: droplet model fit from [40]. The full dots are our results for central collisions. The data and the theoretical results are normalized on the same $A=12$ yield. The error bars represent the statistical uncertainty of our results. See text for detail

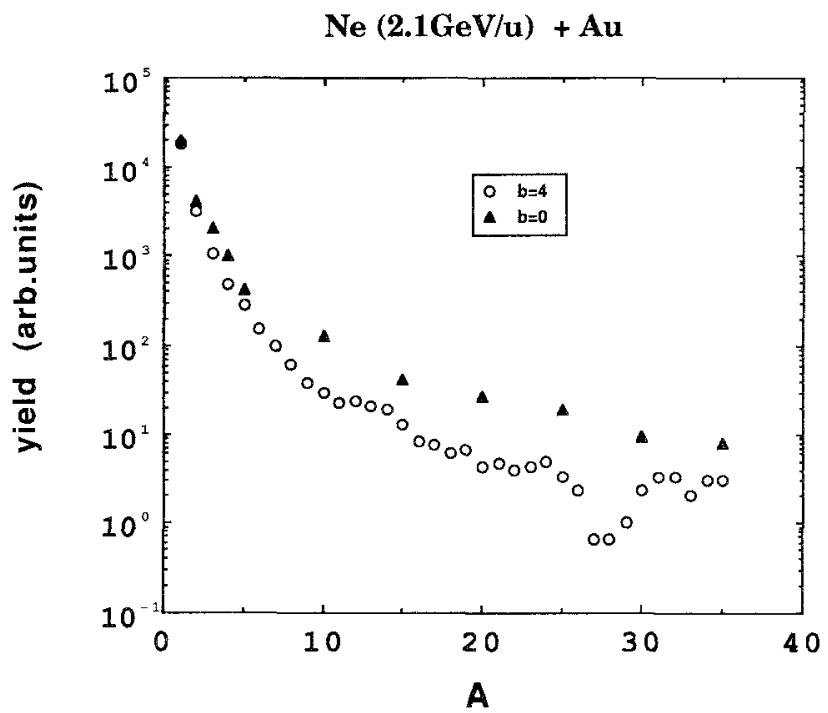

Fig. 7. Mass yield for two impact parameters $(b=0$ and $b=4 \mathrm{fm})$ in $\mathrm{Ne}+\mathrm{Au}$ collisions at $2.1 \mathrm{GeV}$ per nucleon

bution is plotted. Since fission decay, not included in the present calculation, as well as copious evaporation will be suffered by the heavier residual masses, one cannot expect results of Fig. 8 to agree with experiment. Actually, almost all the heavy residues decay by binary fission, but, nevertheless, our calcula- 


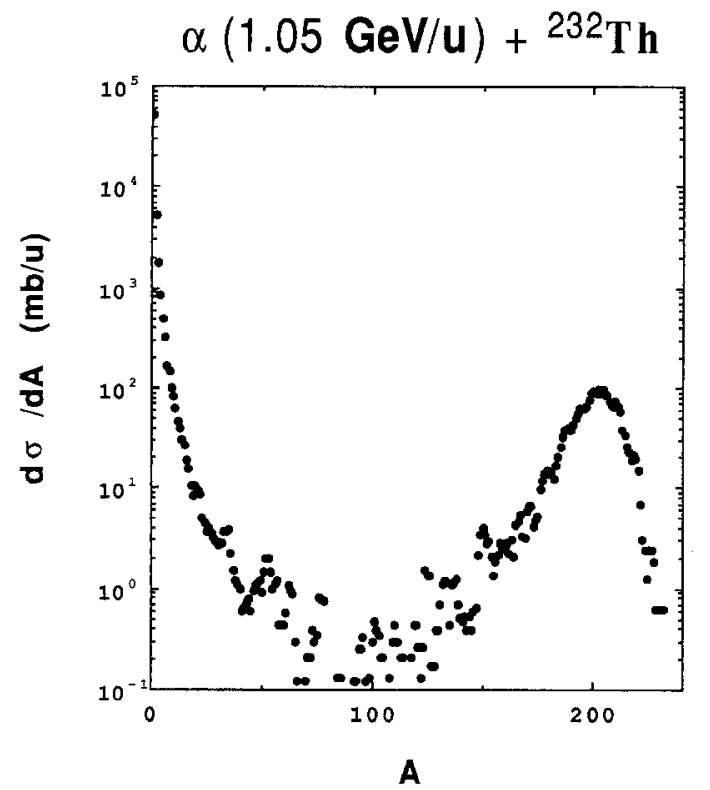

Fig. 8. Primary fragment production cross-section for collisions of $1.05 \mathrm{GeV}$ per nucleon $\alpha$ 's on Th nuclei, as calculated in our model

$\alpha(1.05 \mathrm{GeV} / \mathbf{u})+\mathrm{Th}$

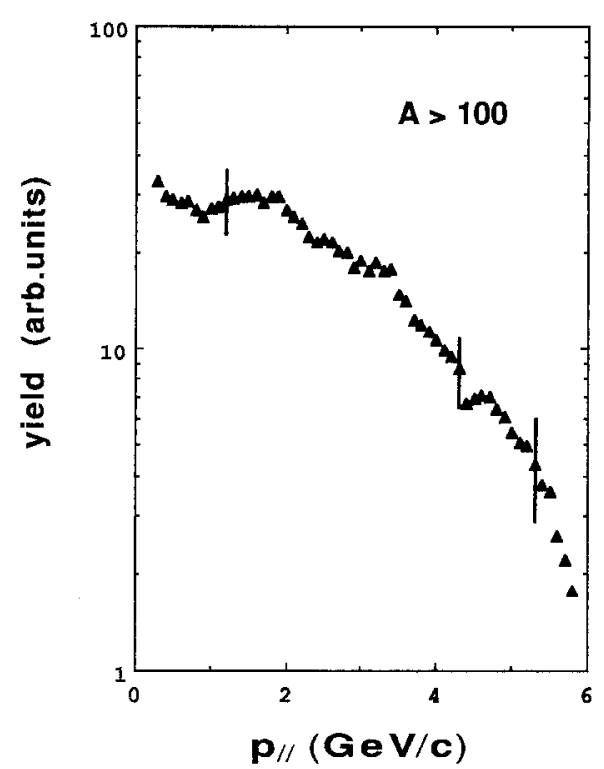

Fig. 9. Longitudinal momentum distribution for $A>100$ fragments produced in collisions between $\alpha$ particles and $\mathrm{Th}$ targets at $1.05 \mathrm{GeV}$ per nucleon. The statistical uncertainty of the calculations is indicated by typical error bars

tion predicts production of IMF's at the level of $10 \mathrm{mb} / \mathrm{amu}$ for $A \approx 20$, in agreement with the measurements of [42], which refer to $800 \mathrm{MeV} / \mathrm{u}$ in fact.

The model is also able to predict several other observables. Unfortunately, for the moment, our esti-

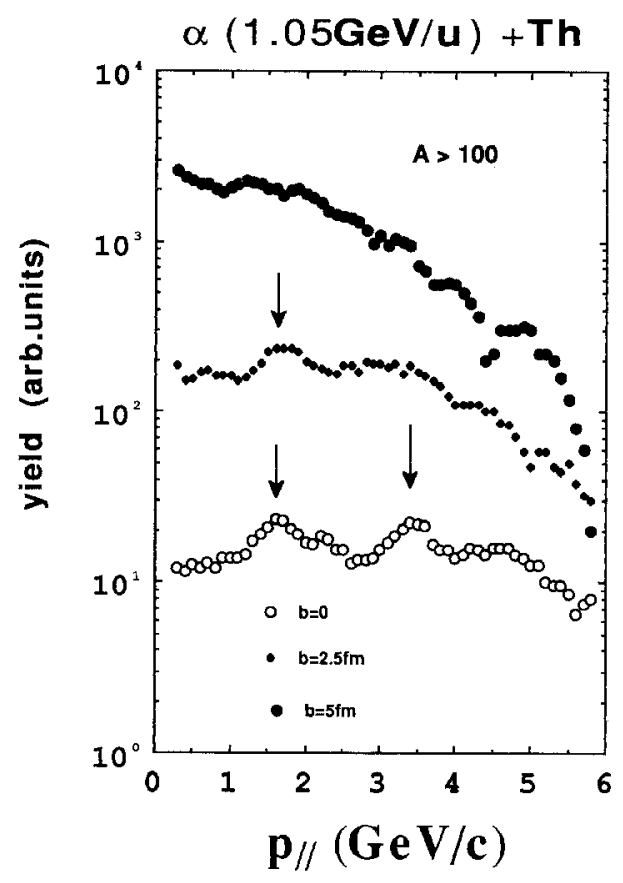

Fig. 10. Comparison between three impact parameters for the distribution of the longitudinal momentum for $(A>100)$ fragments. For the sake of clarity, the curves for $2.5 \mathrm{fm}$ and $5 \mathrm{fm}$ have been multiplied by 10 and 100 , respectively. The arrows indicate the momentum of one or two incident nucleons

mates are not statistically very meaningful. However, to give an idea of the predictive power of the model, we shortly discuss two of them. In Fig. 9 we show the parallel momentum distribution for clusters of mass larger than 100 . One sees that a large fraction of the incident momentum $(6.99 \mathrm{GeV} / \mathrm{c})$ can be transferred to the target (or a large part of it). The momentum transfer does vary very much with the impact parameter as shown by Fig. 10. In our calculation, the transfer of the incident momentum of one $(\sim 1.7 \mathrm{GeV} / \mathrm{c})$ and two nucleons $(\sim 3.4 \mathrm{GeV} / \mathrm{c})$ is clearly visible. For $b=5 \mathrm{fm}$, the transfer of three nucleons perhaps manifests itself. However, we have to remind that we stop the INC calculation at $30 \mathrm{fm} / \mathrm{c}$. In some of the events at least, the momentum is not really spread out and therefore one (or more) very energetic particles will escape if one follows the cascade for a longer time. The parallel momentum distribution is therefore more sensitive to $t_{0}$ than the mass distribution, because the removal of a few nucleons does not really change the latter.

Measurements of $p_{\|}$has been made in $[42,43]$. Unfortunately, the comparison with our calculation is not easy, mainly, but not uniquely, because what is actually measured is the velocity of two fission fragments of a heavy residue. The reconstruction of $p_{\|}$for the primordial heavy fragment is then made assuming binary fission in equal masses, no modification of 
$\alpha(1.05 \mathrm{GeV} / \mathbf{u})+$ Th

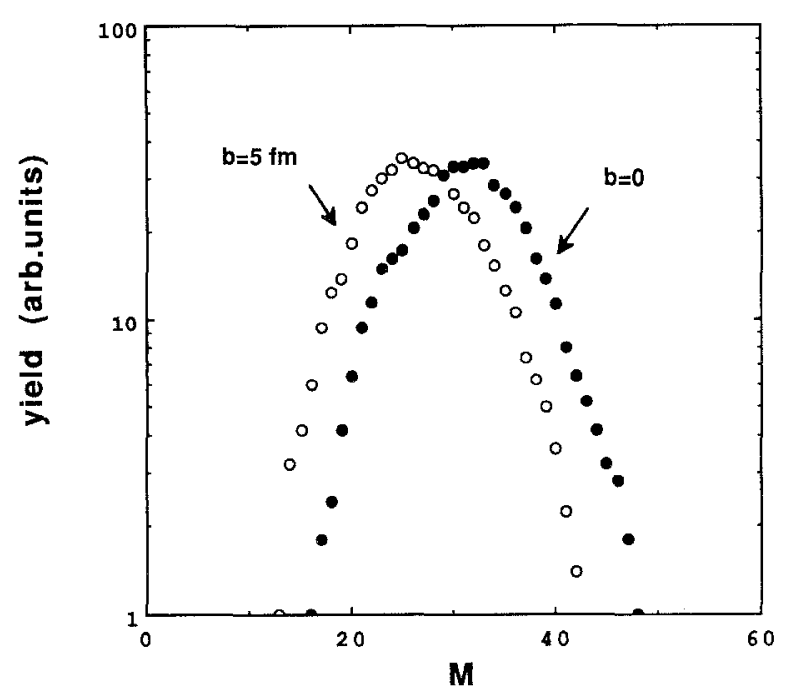

Fig. 11. Fragment multiplicity distributions for events containing at least one heavy fragment $(A>100)$. Collisions between alpha particles and a Th target at $1.05 \mathrm{GeV}$ per nucleon at two impact parameters are considered

fragment velocity by evaporation, and above all the mass of the primordial residue being close to the target mass. Taking account of this and of the fact that the experimental trigger (fission) may differ quite well from our chosen trigger (a heavy residue of mass larger than 100), we can say that we have a fair agreement with the data of [43]. The decrease is however stronger in the experiment. For instance, the data decreases by a factor 10 between 2 and $4 \mathrm{GeV} / \mathrm{c}$, whereas in our prediction there is only a factor 5 .

We can also look a little bit at the fragmentation pattern event by event. For instance, in the same system, there is always (in the limit of our statistics) a heavy $(A>100)$ fragment for $b=5 \mathrm{fm}$. For zero impact parameter, the same statement is almost valid: only $1 \%$ of the events are characterized by the absence of a heavy fragment. In Fig. 11, we show the distribution of the number of fragments (of any mass) for events with at least a fragment with a mass number over 100 . As expected, the average multiplicity decreases with increasing impact parameter, but the shape of the distribution does not seem to change very much.

\section{Conclusion}

We have here presented calculations based on an INC + percolation approach, which look very encouraging. It has furthermore a high predictive power, which has not been fully exploited in this first paper, by far.
We have concentrated on the mass yield, the simplest observable to analyze, but we also discussed the longitudinal momentum of heavy fragments in the $\alpha+T h$ case and the multiplicity distribution in $\alpha+$ Th events with a large fragment. Other quantities can be studied very well in our approach, like energy spectra, moments of the mass distributions, correlations of all kinds, ... The evaluation of some of them will require a large computational effort in order to accumulate sufficient statistics. But this calculation, at least in the $p$-nucleus case, will be possible in the near future.

Our approach can be characterized by a careful description of the interaction in the first step (cascade) and a geometrical (neglecting interactions) treatment of the second step (cluster formation). This contrasts with many approaches which make simplifying assumptions $[16-19]$ for the first step and use similar percolation prescriptions as we did, or with some other approaches [9], which also use a simplified description of the first step and a much more sophisticated treatment of the interactions in the second step. The fact that we obtain good results with our percolation procedure strongly supports the statement that what matters in the fragmentation is the geometrical properties (dispersion, fluctuations, ...) of the system at the end of the (hard collision) first stage, at least for the description of the gross properties of the data. Of course, it would be non-sense to claim that nuclear forces are not responsible for the evolution of the system in the second stage. But we think that only very global properties of the forces are in action in this stage and that many properties of the fragmentation are insensitive to the detail of the forces. One can conjecture that the global properties we allude to can be contained, at least in a phenomenological description, in a single parameter as our parameter $d_{c}$. Systematic study of the value of the parameter $d_{c}$, considered as a phenomenological parameter would allow to determine whether this parameter carries dynamical information and what is the nature of the latter.

In view of the success of many approaches using rather crude descriptions of the first stage, one might wonder whether a detailed treatment of nuclear interactions is necessary for this stage as well. As far as mass yields are concerned, it seems that nuclear forces lead to similar (for different targets and different energies) and fairly random patterns of phase space population and that the almost only relevant feature is the overall density. This may be substantiated by the observation that the mass yield does not depend so much upon the impact parameter. Nevertheless, a detailed treatment of the hard collision process, as ours, is required in order to, at least, predict this overall density. We stress this point, mentioning that excitation functions, like in Fig. 5, are only explained by 
our microscopic calculation, as far as we know. Another example of the importance of an appropriate description of the first stage is provided by the strong dependence upon the impact parameter of the longitudinal momentum distribution (Fig. 10). Evidently, this description is even more important for correlations, which have not been studied very much so far, but which are repeatedly acknowledged as a potential source of information on the fragmentation dynamics.

So, in conclusion, we believe that our approach has a high predictive power, can be very fruitful in the analysis of multifragmentation properties and for a good modelization of "trivial" effects, which can be shortly summarized as "hard scattering plus geometry", so helping to isolate possible unusual behaviour of nuclear systems.

\section{References}

1. Yariv, Y., Fraenkel, Z.: Phys. Rev. C 20, 2227 (1979)

2. Cugnon, J., Mizutani, T., Vandermeulen, J.: Nucl. Phys. A 352 $505(1981)$

3. Cugnon, J.: Nucl. Phys. A 387, 191c (1982)

4. Jasselette, P., Cugnon, J., Vandermeulen, J.: Nucl. Phys. A484, $542(1988)$

5. Cugnon, J.: Nucl. Phys. A 462, 751 (1987)

6. Finn, J.E., Agarwal, S., Bujak, A., Chuang, J., Gutay, L.J., Hirsch, A.S., Minich, R.W., Porile, N.T., Scharenberg, R.P., Stringfellow, B.C., Turkot, F.: Phys. Rev. Lett. 49, 1321 (1982)

7. Warwick, A.I., Wieman, H.H., Gutbrod, H.H., Maier, M.R., Péter, J., Ritter, H.G., Stelzer, H., Weik, F., Freedman, M., Henderson, D.J., Kaufman, S.B., Steinberg, E.P., Wilkins, B.D.: Phys. Rev. C 27, 1083 (1983)

8. Chidwood, C.B., Fields, D.J., Gelbke, C.K., Lynch, W.G., Panagiotou, A.D., Tsang, M.B., Utsunomiya, H., Friedman, W.A.: Phys. Lett. 131 B, 289 (1983)

9. Strack, B., Knoll, J.: Z. Phys. A - Atoms and Nuclei 315, 249 (1984)

10. Gross, D.H.E., Satpathy, L., Ta-chung, M., Satpathy, M.: Z. Phys. A - Atoms and Nuclei 309, 41 (1982)

11. Barz, H.W., Bondorf, J.P., Donangelo, R., Mishustin, I.N., Schulz, H.: Nucl. Phys. A448, 753 (1986)

12. Aichelin, J., Hüfner, J., Ibarra, R.: Phys. Rev. C 30, 107 (1984)

13. Aichelin, J., Peilert, G., Bohnet, A., Rosenhauer, A., Stöcker, H., Greiner, W.: Nucl. Phys. A 448, 437 c (1988)

14. Panagiotou, A.D., Curtin, M.W., Toki, H., Scott, D.K., Siemens, P.J.: Phys. Rev. Lett. 52, 496 (1984)

15. Minich, R.W., Agarwal, S., Bujak, A., Chuang, J., Finn, J.E., Gutay, L.J., Hirsch, A.S., Porile, N.T., Scharenberg, R.P., Stringfellow, B.C., Turkot, F.: Phys. Lett. 118b, 458 (1982)

16. Bauer, W., Post, U., Dean, D.R., Mosel, U.: Nucl. Phys. A 452, $699(1986)$

17. Campi, X., Desbois, J.: Proceedings of the Topical Meeting on Phase Space Approach to Nuclear Dynamics, Trieste, 1985. DiToro, M., Nörenberg, W., Stingari, S. (eds.). Singapore: World Scientific 1986

18. Biro, T.S., Knoll, J., Richert, J.: Nucl. Phys. A 459, 692 (1986)
19. Cerutti, C., Desbois, J., Boisgard, R., Ngô, C., Natowitz, J., Nemeth, J.: Nucl. Phys. A 476, 74 (1988)

20. Ngô, C., Boisgard, R., Desbois, J., Nemeth, J., Barranco, M., Mathiot, J.F.: Nucl. Phys. A 471, 381 c (1987)

21. Ngô, C.: Nucl. Phys. A 488, 233c (1988)

22. Barz, H.W., Bondorf, J.P., Schulz, H.: Nucl. Phys. A 462, 742 (1987)

23. Porile, N.T., Bujak, A.T., Carmony, D.D., Chung, Y.H., Gutay, L.J., Hirsch, A.S., Mahi, M., Paderewski, G.L., Sangster, T.C., Scharenberg, R.P., Stringfellow, B.C.: Nucl. Phys. A 471, 149c (1987)

24. Campi, X.: J. Phys. A 19, L917 (1986)

25. Gutbrod, H.H., Sandoval, A., Johansen, P.J., Poskanzer, A.M., Gosset, J., Meyer, W.G., Westfall, G.D., Stock, R.: Phys. Rev. Lett. 37, 667 (1976)

26. Guylassy, M., Frankel, K., Remler, E.A.: Nucl. Phys. A 402, 596 (1983)

27. Bertsch, G., Cugnon, J.: Phys. Rev. C 24, 2514 (1981)

28. Lemaire, M.C., Nagamiya, S., Schnetzer, S., Steiner, H., Tanihata, I.: Phys. Lett. 85 B, 38 (1979)

29. Bauer, W., Dean, D.R., Mosel, U., Post, U.: Phys. Lett. 150 B, $53(1985)$

30. Desbois, J.: Nucl. Phys. A 446, 724 (1987)

31. Cugnon, J., Kinet, D., Vandermeulen, J.: Nucl. Phys. A 379, 553 (1982)

32. Cugnon, J., Lemaire, M.C.: Nucl. Phys. A 489, 787 (1988)

33. Dijkstra, E.W.: Numer. Mat. 1, 269 (1959)

34. Dorfan, J.: Z. Phys. C - Particles and Fields 7, 349 (1981)

35. Bertsch, G., Siemens, P.J.: Phys. Lett. 126 B, 9 (1983)

36. Cugnon, J.: Phys. Lett. 135 B, 374 (1984)

37. Porile, N.T., Bujak, A.T., Carmony, D.D., Chung, Y.H., Gutay, L.J., Hirsch, A.S., Mahi, M., Paderewski, G.L., Sangster, T.C., Scharenberg, R.P., Stringfellow, B.C.: Nucl. Phys. A 471, 149 c (1987)

38. Mahi, M., Bujak, A.T., Carmony, D.D., Chung, Y.H., Gutay, L.J., Hirsch, A.S., Paderewski, G.L., Porile, N.T., Sangster, R.P., Stringfellow, B.C.: Phys. Rev. Lett. 60, 1936 (1988)

39. Sangster, T.C., Bujak, A.T., Carmony, D.D., Chung, Y.H., Gutay, L.J., Hirsch, A.S., Mahi, M., Paderewski, G.L., Porile, N.T., Scharenberg, R.P., Stringfellow, B.C.: Phys. Lett. 188 B, 29 (1987)

40. Goodman, A.L., Kapusta, J.I., Mekjian, A.Z.: Phys. Rev. C 30, $851(1984)$

41. Cugnon, J.: In: Heavy ion collisions. Bonche, P. et al. (eds.), p. 209. New York: Plenum Press 1986

42. Klotz. Engmann, G., Oeschler, H., Kankeleit, E., Cassagnou, Y., Conjeaud, M., Dayras, R., Harar, S., Mostefai, M., Legrain, R., Pollacco, E.C., Volant, C.: Phys. Lett. 187 B, 245 (1987)

43. Klotz. Engmann, G., Oeschler, H., Stroth, J., Kankeleit, E., Cassagnou, Y., Conjeaud, M., Dayras, R., Harar, S., Legrain, R., Pollacco, E.C., Volant, C.: Nucl. Phys. A 499, 392 (1989) and private communication

J. Cugnon

Physique Nucléaire Théorique

Institut de Physique au Sart Tilman

Université de Liège

Bâtiment B.5

B-4000 Liège 1

Belgium

C. Volant

$\mathrm{DPhN}-\mathrm{Be}$

CEN Saclay

F-91191 Gif-sur-Yvette Cedex

France 